Témoigner Témoigner. Entre histoire et mémoire

Getuigen Revue pluridisciplinaire de la Fondation Auschwitz

$126 \mid 2018$

Questions sur l'avenir du travail de mémoire

\title{
En Italie du nord, sur les traces de la résistance au fascisme. Trois mémoriaux en souvenir des victimes
}

Yannik van Praag

\section{(2) OpenEdition \\ Journals}

Édition électronique

URL : https://journals.openedition.org/temoigner/7420

DOI : $10.4000 /$ temoigner.7420

ISSN : 2506-6390

Éditeur :

Éditions du Centre d'études et de documentation Mémoire d'Auschwitz, Éditions Kimé

Édition imprimée

Date de publication : 2 avril 2018

Pagination : 138-144

ISBN : 978-2-930953-06-9

ISSN : 2031-4183

Référence électronique

Yannik van Praag, «En Italie du nord, sur les traces de la résistance au fascisme. Trois mémoriaux en souvenir des victimes », Témoigner. Entre histoire et mémoire [En ligne], 126 | 2018, mis en ligne le 24 janvier 2022, consulté le 04 février 2022. URL : http://journals.openedition.org/temoigner/7420 ; DOI : https://doi.org/10.4000/temoigner.7420 


\section{Site mémoriel}

\section{EN ITALIE DU NORD, SUR LES TRACES \\ DE LA RÉSISTANCE AU FASCISME. TROIS MEMORIAUX EN SOUVENIR DES VICTIMES}

L'ASBL Ami, entends-tu ? et l'ANPI (Association nationale des Partisan italiens) ont organisé fin septembre 2017 un voyage d'études en Italie du Nord sur les traces de la résistance au fascisme. L'objectif principal de ce périple était dévaluer et de finaliser, sur le terrain, un voyage qui sera proposé à des groupes dadolescents et de jeunes adultes afn de les sensibiliser à lhistoire du La Fondation Auschwitz y a participé.

Nous présentons ici trois mémoriaux élevés en hommage aux victimes de cette violence. Ils rappellent les ravages du fascisme mais aussi que c'est une véritable guerre civile qui a embrasé l'Italie mussolinienne à son crépuscule. résistance à travers le pays. Sil garde les plaines, on nes centes dibains et des planes, on ne peut en dire autant d'acis, d'ou des groupes de partians prép d d tes gent leurs ón La résistet lancent leurs operations. La résistance ancée aurait compté quelque 250000 conbattants, auxquels d'hom ajouter les dizaines de nilliers varent d'axiliares et de soutien, dar les villes et les cas et de soutien, dans Jextrême violencedela

Lextrem de ces réalités. Allemands et milices partisans appent mon senlenent les partis

LE MASSACRE DE LA BENEDICTA

Dans le parc naturel de Capanne di

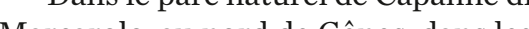
Apennins, aux limites du Piémont de la Ligure se dressent les runes l'abbing labbaye bécictine Cest la et dans came pétré en avil 1944 le ples grand morsacre de partisans en Italie acours de la Seconde Guerre mondiale.

Dès la proclamation de la RSI, des Dilaires réfractaires et dejeunes gens de la région qui refusent de prendre

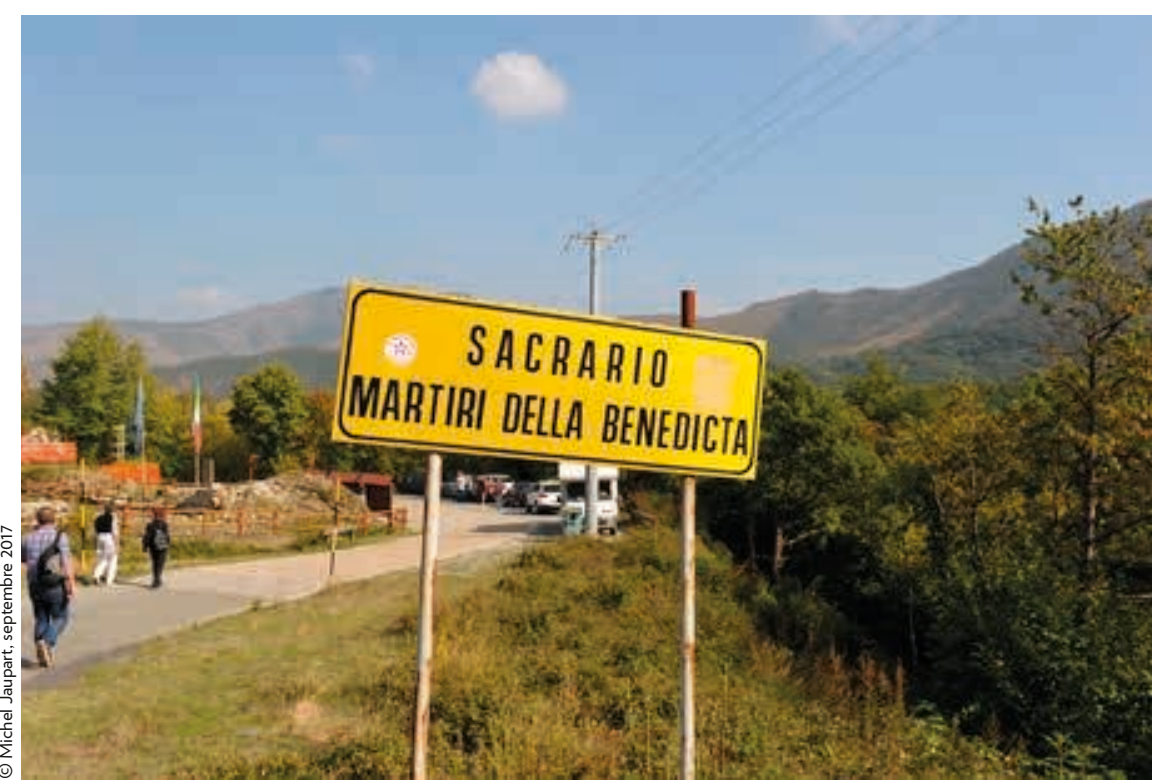

Entrée du site de

mis en minorité par le Grand Consei fasciste le 24 juillet 1943. Il est arrêté le lendemain et assigné à résidence au Campo Imperatore, dans les Abruzzes. Le 8 septembre, la reddition italienne est rendue publique et, le 12, Mussolin est exfiltré par un commando allemand. Il est placéà la tête d'un gouvernement qui conservera le contrôle sur le nord du pays, soutenu par d'importants continents militaires allemands.

Dans les faits, ce ne sont plus Mussolini et ses séides qui décident, mais bien Albert Kesselring (plénipotentiaire et plénipotentiaire civil) et Karl Wolff
Benedicta

(2) Préface de Bernard
Droz de la monographie de Claudio Pavone, Une

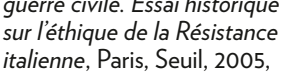
p. 8 . plus brutaux du fascisme it

Sicile durant l'été 1943, Mussolini est 


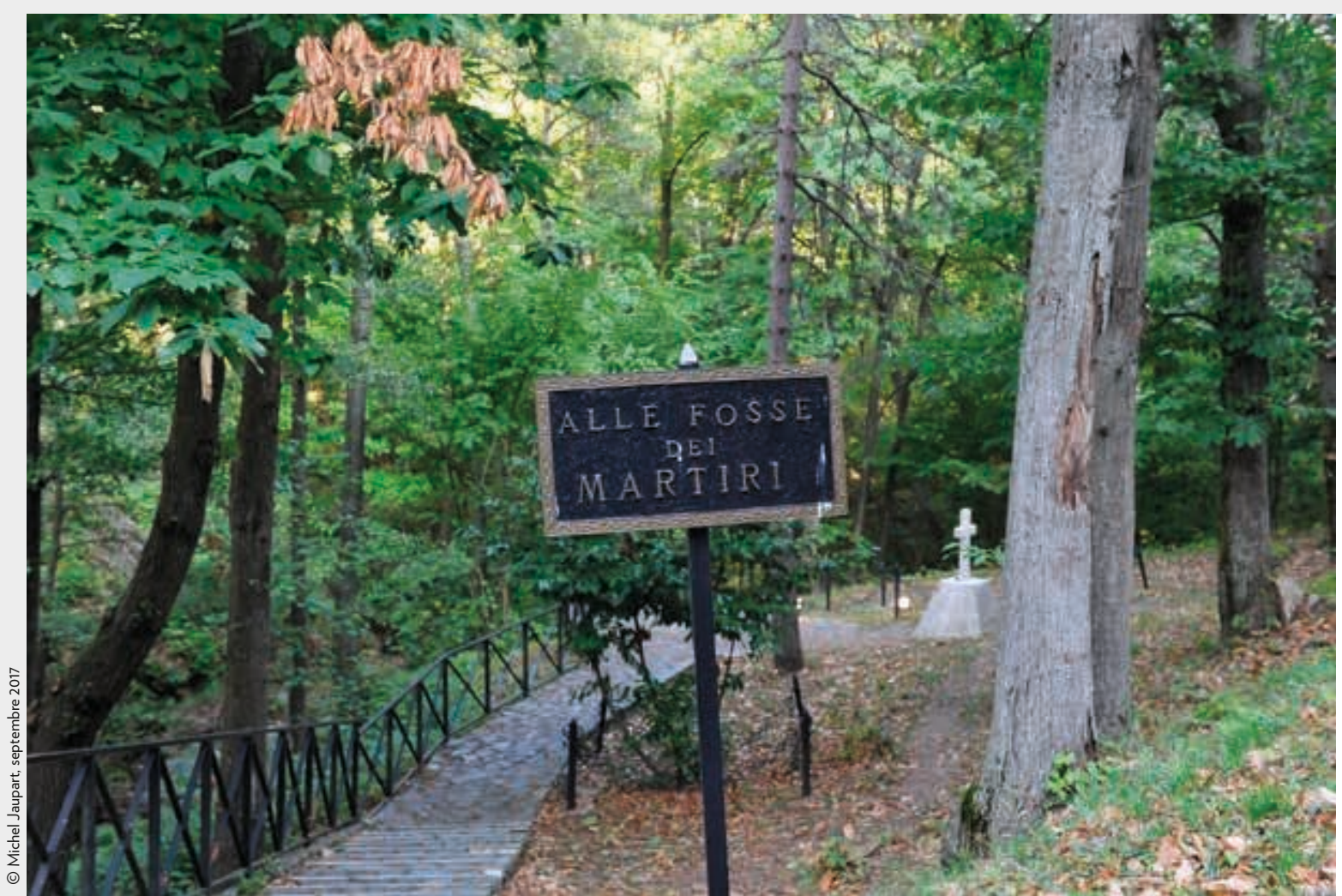

4 Chemin menant

martyrs, abbaye de
la Benedicta

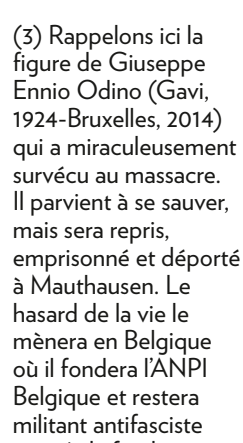

•. les armes sous son drapeau se réfugient dans ces collines. Leur nombre s'accroît rapidement pour approc
700 hommes au printemps 1944 .

Entrele 6et le 11 avril 1944,2500 soldats dela 356 e division de la Wehrmach dats dela $356^{\circ}$ divisiondela Wehrmacht soutenus par près de 400 fascistes italiens bloquent les routes et lancent loffensive contre des partisans qui se dure plus surs jours Lespli. La traque dure plusins jous Lespartisans sont sinés froid loms des coǹ ats ou assassinés froidement après avoir éte faits prisonniers. Les assaillants mettent le

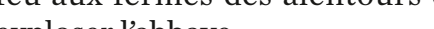
Près de 150 partisans sont fusil-
lés quelque temps plus tard, d'autres tombent au combat. Environ 400 sont capturés et déportés, pour la plupart à reviendront pas.

À la fin de la guerre, l’abbaye est laissée en ruine, abandonnée. C'est la population locale qui perpétuera la mémoire il de mémoire avec les école.

Aujourd'hui, à quelques dizaines de mètres des ruines de l'abbaye, se dressent des dalles de labbaye, se mage aux partisans assassinés juste en contre-haut des fosses où le, juste furent entasćs. Ie tout lons corps vaste ensemble mémoriel. Un bâtiMauthausen, dont près de la moitié ne s victimes, engageant notamment ment en construction jouxte également l'abbaye Il accueillera un centre de documentation destiné à réunir les documents et archives concernant la destance dans légion et actuelleNos hôtes italiens qui nous ont fait récit de ces ćnéne qui nous ont fait lie res even toute limportáce de transmettre aux nouvelles generrations ce qu'est le fascisux qui ont pris les armes animes résister. Ils rappellent auss pour lui quister. Is rappellent aussi que ceux qui ont assassiné à a mitrailleuse les d'ensevelir leurs corps dar cinq avant dens

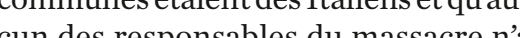
passé un seul jour en

LA CASA CERVI

Le 25 novembre 1943, Alcide Cerviet ses sept fils - Gelindo (né en 1901), Antenore (1906), Aldo (1909), Ferdinando (1911), Agostino (1916), Ovidio (1918), Ectore (1921) (1921), cont arrêtés dans lan fer slcide perviendra miracu faniliale. Alcide parviendra miraculeusement à sechaper, mas sé

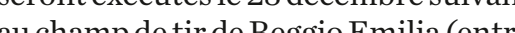
Parine detire Parme et Modène)

LaCasa Cervi,est devenueun musée un lieu emblématique de mémoire de l'antifascisme en Italie de mémoire de lantifisme, puisquil Cocsupe authentique, puisqu il occupe la ferme an poring a x potes de leur do stine agricole de seze hectares, entre les cons '’histoire drapegique d

Ler dervi frappe par son intensité. Dès le porch

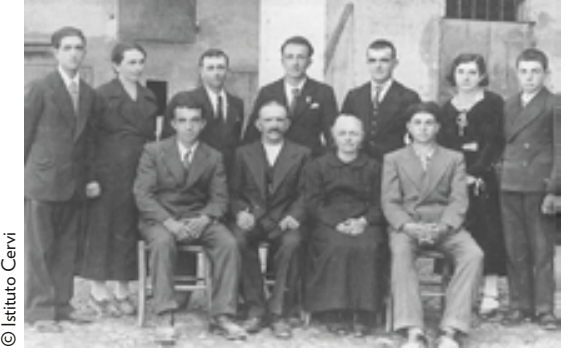

photo de famille de ces modestes paysans dont tous les fils ont étéfroidement assassinés, sans procès.

Nous ne sommes pas véritablement ici dans le récit d'une résistance héroïque de partisans armés, organisés, et au socle idéologique solide. La famille Cervi est humble, rurale et catholique. Elle n'est pas particulièrement engagée politiquement, à l'exception d'Aldo, intellectuel et militant communiste dont son père dira plus tard « Aldo m'a donné le peu d'intelligence politique que j'ai. »

L'engagement des frères Cervi commence véritablement lors de l'éviction de Mussolini en juillet 1943. La foule envahit alors les places publiques, les Cervi et d'autres familles participent à l'allégresse en apportant à Campegine de copieux plats de pâtes qu'ils distribuent gratuitement ${ }^{4}$.

Malheureusement, l'allégresse sera de courte durée. Les fils Cervi prennent le maquis, mais à l'arrivée de l'hiver, manquant de ressources, ils sont contraints de redescendre dans lavallée, où ils sont capturés par les fascistes qui pillent la ferme familiale.

La scénographie du musée mêle intelligemment la résistance antifascisteà l'histoire de la ruralitéitalienne et sa modernisation. En effet, la ferme est $\diamond$ La famille Cervi peu avant le drame

(4) En hommage àce
geste qui symbolise résistance populaire,
la tradition purdure aujourd'hui : une
enorme spaghettata est organisé chaque

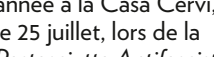
Pastasciutta Antifascista 


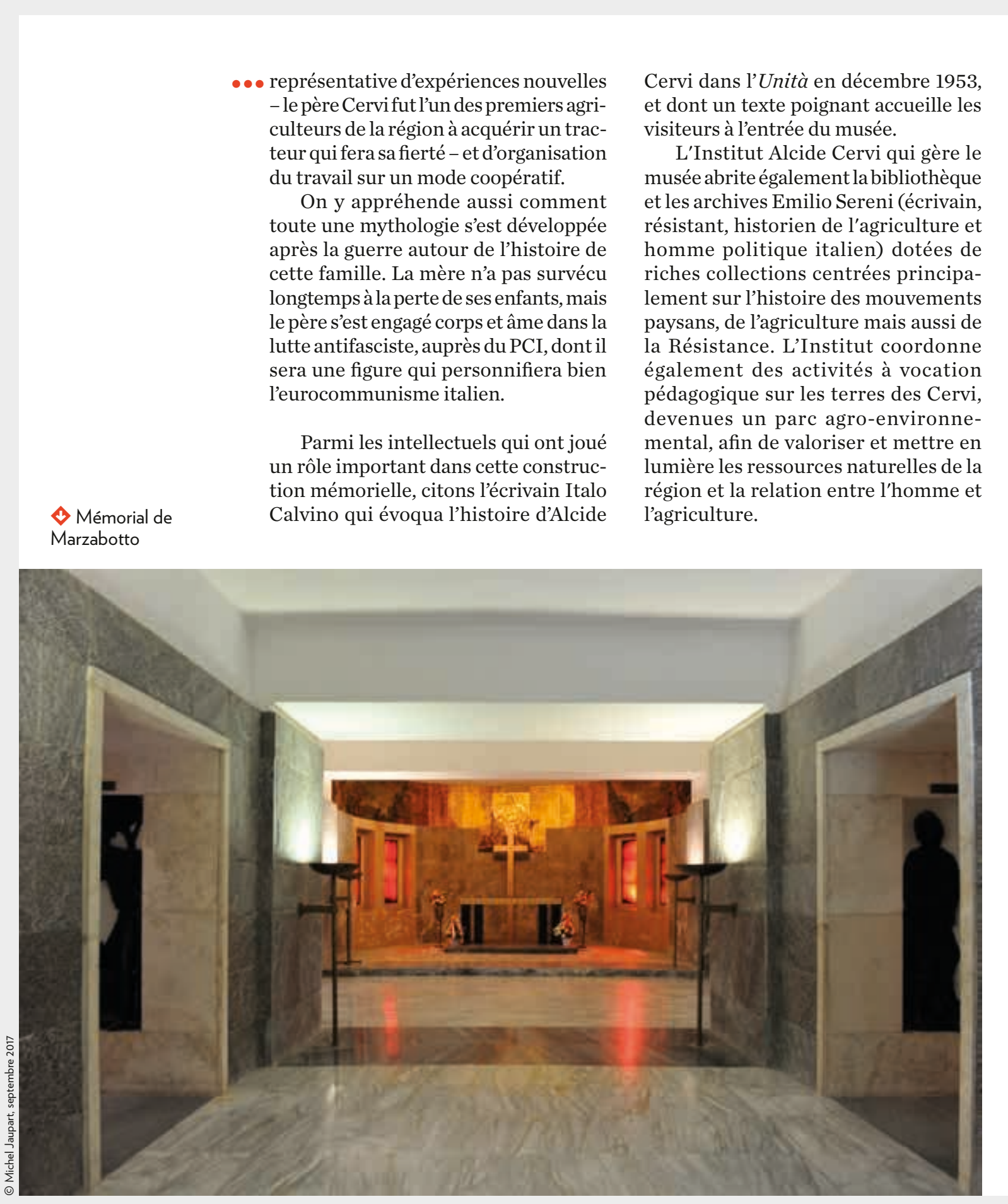

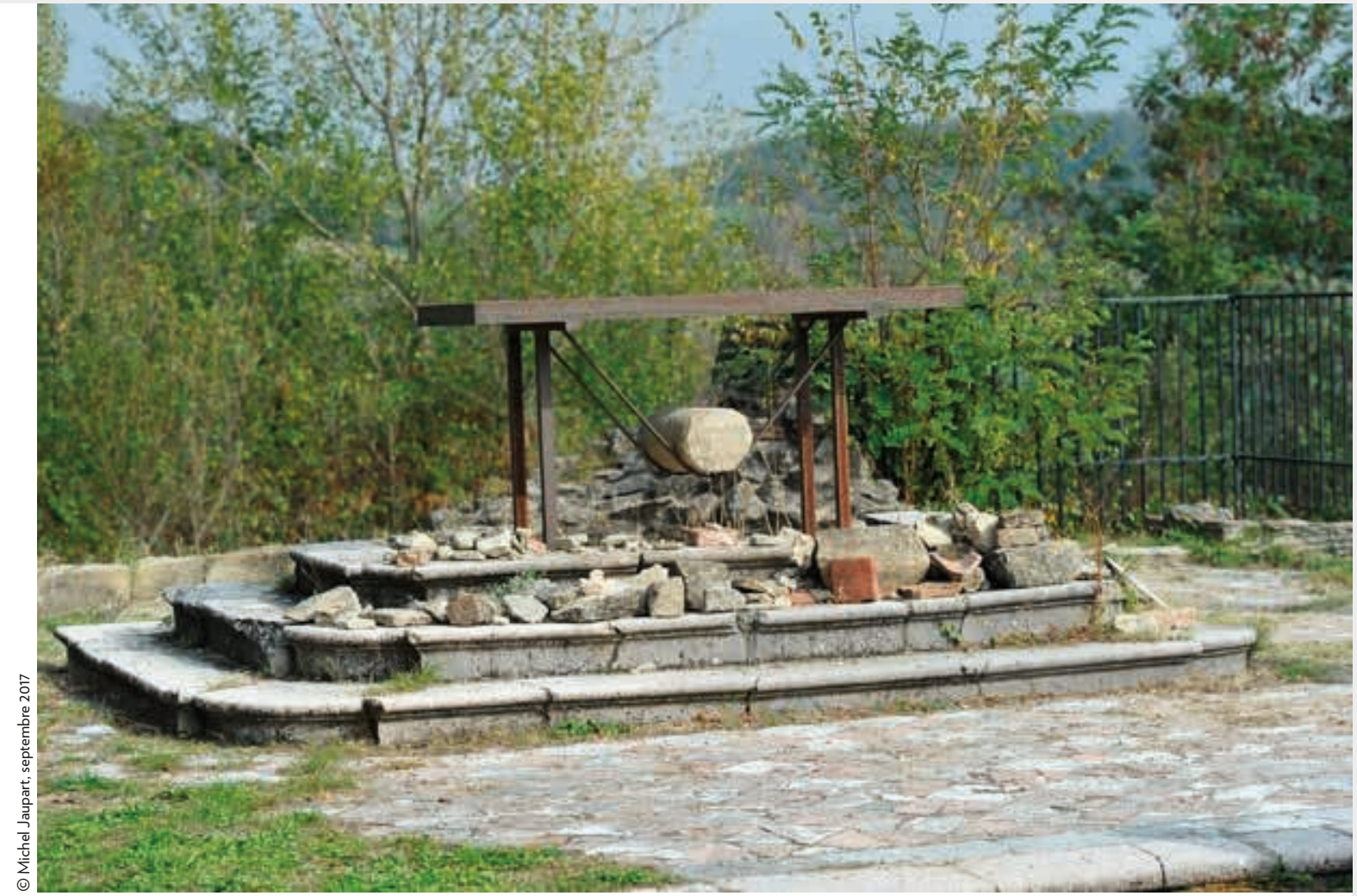

MONTE SOLE, ORADOUR ITALIEN Septembre 1944, les troupes allemandes sont repoussees par les Alliés nins, où elles sont égalemgdes Apentés, ac elles sonte égalenent confronLe SS Gruppenführer M.

Le SS Gruppenführer Max Simon ordonne une operration de «nettoyage Sole, audde Bontagneuxde Monte Sole, aus de Bologne, point dancrage decomburistis de combattants issus de diferents hor zons politiques et philosophiques.

Lassaut est done sur un vaste perilàtre délimité par et Gizzana Morandi. Paro, Monzuno et Grizzana Morandi. Parmi les forces $16^{\mathrm{e}}$ SS Panzergrenadier Division Reichs-

$\diamond$ Coeur de l'église en Walter Reder, sinistrement connu, notamment pour son action dans la liquidation du ghetto de Varsovie au de toute la population du village de Sant'Anna di Stazzema (Toscane) en août 1944.

Les « opérations » durent jusqu’au 5 octobre et ne se limitent pasà la chasse sont les populations civiles, coincées à l'intérieur du périmètre. Certains villages sont rasés et leurs habitants systéführer-SS, sous le commandement de printemps 1943 et pour le massacre aux partisans. Les principales victimes matiquement massacres. La violenceest 
(5) « Le pays étant gouverné dès 1947 par des forces politiques qui n'avaient aucun intérêt à exalter un héritage transmis par des partis de gauche (en particulier le Parti communiste et le Parti d'action) désormais dans I'opposition », Ersilia Alessandrone Perona, «Les musées de la résistance en Italie ", in Daniel Grange et Dominique Poulot, L'esprit des lieux. Le patrimoine et la Cité, Grenoble, PUG, 1997, p. 447-453.

(6) Rappelons cependant l'existence du musée de la Résistance de Belgique rue Van Lint, à Anderlecht (http://www.museumresistance.be/). $\bullet$ de civils (femmes, enfants, vieillards) dans les cimetières de San Martino et de Casaglia. Nous ne discuterons pas ici du nombre total des victimes. Près de 800 certainement, peut-être près du double. Ce fut le plus important massacre de civils perpétré par les nazis en Europe occidentale.

Des stèles et des monuments ont été placés sur les différents lieux de ces crimes dans le parc régional de Monte Sole.

La petite ville de Marzabotto est, quant à elle, reconnue par l'ONU comme Città messaggera di pace (ville messagère de la paix) et son imposant mémorial rend non seulement hommage à tous ceux qui sont tombés lors de ces journées de septembre-octobre 1944, mais à toutes les villes martyres à travers le monde.

\section{CONCLUSION}

Ces mémoriaux témoignent de la brutalité de la répression sous la RSI. Ils ne représentent qu'une part infime du déferlement de violence qui a frappé l'Italie entre 1943 et 1945, du massacre des Fosses ardéatines à la déportation des Juifs italiens (qui débute avec l'établissement de la RSI).

Ce qui interpelle également, c'est la place qu'occupe l'histoire de la Résistance dans la mémoire italienne. La perpétuation de celle-ci est très différente des cas belges et français. Alors qu'en France, cette histoire a été véritablement institutionnalisée, entre autres à travers un grand nombre de musées, elle a été, pour des raisons qui appartiennent au contexte politique d'aprèsguerre $^{5}$, transmise davantage grâce à des initiatives privées, associatives, locales, ponctuelles.
Des associations telles que L'ANPI (qui revendique aujourd'hui 120000 membres) s'attachent à jouer un rôle de relais entre les générations. Elles œuvrent à donner du sens à ces récits pour promouvoir un authentique engagement citoyen auprès des plus jeunes.

On n'a pas construit cette mémoire en Belgique. Bien sûr, notre histoire n'est pas la leur, mais comme le faisait remarquer la professeure Anne Morelli durant notre séjour, tout le monde connaît l'histoire des frères Cervi en Italie. Qui, hors de quelques cercles restreints est capable de citer une figure marquante de la Résistance belge ${ }^{6}$ ? I

Yannik van Praag 